\title{
Artificial Neural Networks (ANN) Approach to Modelling of Selected Nitrogen Forms Removal from Oily Wastewater in Anaerobic and Aerobic GSBR Process Phases ${ }^{\dagger}$
}

\author{
Piotr Ofman * and Joanna Struk-Sokołowska \\ Department of Environmental Engineering Technology and Systems, Faculty of Civil and Environmental \\ Engineering, Bialystok University of Technology, Bialystok 15-341, Poland; j.struk@pb.edu.pl. \\ * Correspondence: p.ofman@pb.edu.pl; Tel.: +48-571-443-155 \\ + Presented at Innovations-Sustainability-Modernity-Openness Conference (ISMO'19), Bialystok, Poland, \\ 22-23 May 2019.
}

Published: 13 June 2019

\begin{abstract}
The paper presents artificial neural network models approximating concentration of selected nitrogen forms in wastewater after sequence bath reactor with aerobic granular activated sludge (GSBR) anaerobic and aerobic phase. Developed models reflected all the changes in concentration of studied nitrogen forms $(\mathrm{r}=0.996-0.999)$. In models approximating Total $\mathrm{N}$ and $\mathrm{N}$ $\mathrm{NH}_{4}$, variable most influencing calculations was nitrogen form at the beginning of anaerobic or aerobic phase.
\end{abstract}

Keywords: GSBR; ANN model; nitrogen removal

\section{Introduction}

The most common form of activated sludge is flocked sludge. Most of the papers refer to the description of phenomena occurring during wastewater treatment with its use. However, in recent years, more and more interest has been directed towards the aerobic granular activated sludge. According to the definition presented by International Water Association, this form of activated sludge is referred to as aggregates of a microbiological nature, not coagulating under the influence of shear forces and characterized by a lower sedimentation time compared to flocked sludge [1]. Many researchers presented both the ability of this activated sludge form in removing anthropogenic pollution from wastewater, as well as high tolerance for unfavorable conditions for wastewater treatment process [2]. The contamination of petroleum products, including oils and lubricants, are relatively onerous from the point of biological wastewater treatment. These substances limit the gas exchange of microorganisms involved in wastewater treatment processes. It should be emphasized that despite numerous researches devoted to the application possibilities of aerobic granular activated sludge, attempts to model the wastewater treatment process with its use are rarely undertaken. This results from the fact that aerobic granular activated sludge technology is relatively new, hence there is a lack of sufficiently long research on objects operated in real conditions to create the basis for software describing the distribution kinetics of individual pollutants [3]. For this reason, the use of artificial neural networks can closer define activated sludge technological parameters conditioning the degradation processes of pollutants using aerobic granules.

The aim of the work was to develop artificial neural networks models to predict the concentration of selected nitrogen forms in oily wastewater after anaerobic and aerobic phase of sequence bath reactor with aerobic granular activated sludge (GSBR) reactor with fluctuating biological oxygen demand (BOD) load in wastewater flowing into the reactor and determination of 
the most important activated sludge technological parameters for individual nitrogen forms concentration approximation in anaerobic and aerobic phase.

\section{Materials and Methods}

Laboratory GSBR reactor was made of reinforced polyethylene (HDPE). The air was supplied by an aerator with a capacity of $550 \mathrm{dm}^{3} \cdot \mathrm{h}^{-1}$. The active volume of the reactor was $15 \mathrm{dm}^{3}$. The contents of the reactor were mixed using a stirrer with a rotational speed of 70 RPM. The decanting coefficient in the experiment was 0.33 for a single cycle. Duration of the unit phases of the SBR reactor, operation of air pumps and mixers was coordinated with the SIMENS programmable controller, model LOGO!, type 230RC. Studies were carried out in a 12-hour operation cycle, with 30 minutes filling phase duration, 90 minutes of mixing (anaerobic) phase, 540 minutes of mixing and aeration (aerobic) phase, 60 minutes of sedimentation phase and 30 minutes of decantation phase [4].

Wastewater used in studies was prepared from peptone $\mathrm{K}\left(0.113-4.520 \mathrm{~g} \cdot \mathrm{dm}^{-3}\right)$, enriched dry broth $\left(0.076-3.040 \mathrm{~g} \cdot \mathrm{dm}^{-3}\right), \mathrm{NH}_{4} \mathrm{Cl}\left(0.010-0.400 \mathrm{~g} \cdot \mathrm{dm}^{-3}\right), \mathrm{NaCl}\left(6.59 \pm 0,01 \mathrm{~g} \cdot \mathrm{dm}^{-3}\right), \mathrm{CaCl} \cdot 6 \mathrm{H}_{2} \mathrm{O}$ $\left(0.004-0.160 \mathrm{~g} \cdot \mathrm{dm}^{-3}\right), \mathrm{MgSO}_{4} \cdot 7 \mathrm{H}_{2} \mathrm{O}\left(0.001-0.040 \mathrm{~g} \cdot \mathrm{dm}^{-3}\right), \mathrm{KH}_{2} \mathrm{PO}_{4}\left(0.008-0.320 \mathrm{~g} \cdot \mathrm{dm}^{-3}\right)$ and $\mathrm{K}_{2} \mathrm{HPO}_{4}$ $\left(0.020-0.800 \mathrm{~g} \cdot \mathrm{dm}^{-3}\right)$ and oily substances $\left(0.16 \mu \mathrm{l} \cdot 5 \mathrm{dm}^{-3}\right)$. An oily wastewater solution was prepared on the basis of naphtha (60\%), burned-out car oil (30\%) and petroleum oil $(10 \%)$.

Quantitative analysis of $\mathrm{N}-\mathrm{NH}_{4}, \mathrm{~N}-\mathrm{NO}_{3}$, Total $\mathrm{N}$ was performed by UV-VIS spectrophotometric method with Merck UV-VIS Pharo 300 spectrophotometer. The dedicated reagents from Merck were used for individual parameters determination. Samples preparation for analysis was carried out in accordance with the methodology appended by the manufacturer. The considered technological parameters of the activated sludge were calculated based on the following equations:

Activated sludge index:

$$
\mathrm{ASI}=\frac{\mathrm{V}_{\mathrm{os}_{30}}}{\mathrm{G}}
$$

Activated sludge retention time:

$$
\mathrm{SRT}=\frac{\mathrm{n} \cdot \mathrm{V}_{\mathrm{RP}} \cdot \mathrm{Z}_{\mathrm{X}}}{\Delta \mathrm{G}}
$$

Activated sludge age:

BOD load of activated sludge mass:

$$
\mathrm{ASA}=\mathrm{SRT} \cdot \frac{\mathrm{t}_{\mathrm{R}}}{\mathrm{t}_{\mathrm{C}}}
$$

$$
A^{\prime}=\frac{Q_{d} \cdot S_{B O D}}{n \cdot V_{R P} \cdot Z_{R P}} \cdot \frac{t_{R}}{t_{C}}
$$

The multilayer perceptron (MLP) artificial neural networks were used for concentrations prediction of individual nitrogen forms. Input variables in each network constituted the concentration of the approximated nitrogen form, the BOD load, and activated sludge basic technological parameters, including activated sludge index, activated sludge retention time, and activated sludge age. Best algorithm was selected form sampling among 500 networks. In all networks, the training algorithm Broyden-Fletcher-Goldfarb-Shanno was used, and it was assumed that the maximum number of epochs will be 200. The aim of such an approach was to limit the overfilling risk of artificial neural network (ANN). As the error function in all algorithms, the sum of squares was assumed. Estimation error values were determined in accordance with the following equation:

$$
\mathrm{SOS}=\sum_{\mathrm{i}=1}^{\mathrm{n}}\left(\mathrm{x}_{\mathrm{i}}-\overline{\mathrm{x}_{\mathrm{i}}}\right)^{2}
$$

In all the ANN models in the hidden and initial layer, linear function, hyperbolic tangent, logistic, exponential and sine as the activation functions of the layer were considered. ANN models were developed using the Statistica version 13.1 software working on the Windows 10 platform, 696 measurement results were used for modeling. Table 1 presents the network topology, training algorithm, error function, and activation function of neurons in the hidden and input layer in developed ANN models. 
Table 1. Sensitivity analysis and artificial neural networks (ANNs) matching to observed values.

\begin{tabular}{|c|c|c|c|c|c|c|c|c|}
\hline \multirow{2}{*}{$\begin{array}{c}\text { ANN } \\
\text { Topology }\end{array}$} & \multirow{2}{*}{ Estimated $\mathrm{N}$ form } & \multicolumn{5}{|c|}{ Variables rank } & \multirow{2}{*}{$\begin{array}{c}\text { ANN Prediction } \\
\text { Quality (r) }\end{array}$} & \multirow{2}{*}{$\begin{array}{l}\text { ANN } \\
\text { Error }\end{array}$} \\
\hline & & BOD Load & N Form & ASA & ASI & SRT & & \\
\hline MLP 5-6-1 & $\mathrm{N}-\mathrm{NO}_{3}$ in anaerobic phase & 82.37 & 71.84 & 3.33 & 1.68 & 1.20 & 0.999 & 1.373 \\
\hline MLP 5-7-1 & $\mathrm{N}-\mathrm{NH}_{4}$ in anaerobic phase & 22.77 & 1215.16 & 5.47 & 3.85 & 7.70 & 0.999 & 0.061 \\
\hline MLP 5-9-1 & Total $\mathrm{N}$ in anaerobic phase & 84.34 & 87.47 & 3.06 & 3.69 & 1.06 & 0.999 & 2.757 \\
\hline MLP 5-9-1 & $\mathrm{N}-\mathrm{NO}_{3}$ in aerobic phase & 46.80 & 46.42 & 6.97 & 1.36 & 2.97 & 0.998 & 1.115 \\
\hline MLP 5-4-1 & $\mathrm{N}-\mathrm{NH}_{4}$ in aerobic phase & 59.43 & 69.98 & 7.23 & 3.60 & 4.49 & 0.998 & 0.139 \\
\hline MLP 5-3-1 & Total $\mathrm{N}$ in aerobic phase & 30.37 & 30.75 & 2.58 & 2.12 & 2.67 & 0.996 & 3.950 \\
\hline
\end{tabular}

\section{Results}

Developed ANN models showed a good fit in relation to the observed values (Table 1). The prediction quality for individual models ranged from 0.996 to 0.999 depending on the nitrogen form and GSBR process phase. It was observed that the approximated value of $\mathrm{N}-\mathrm{NO}_{3}$ in anaerobic and aerobic phase was depending in most form BOD load, while concentration of $\mathrm{N}^{-\mathrm{NH}_{4}}$ and total nitrogen was influenced by the concentration of this nitrogen form in wastewater. The developed models showed a good match to the observed values (Figures 1-6). Both in the anaerobic and aerobic phase, ANN models reflected the general changes trend in individual concentrations of studied nitrogen forms. It should be noted that the values approximated by the networks matched most of the extreme values that do not fit directly into the trend of changes.

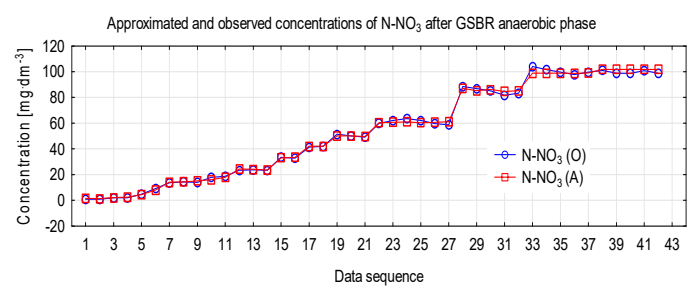

Figure 1. Approximated and observed concentrations of $\mathrm{N}-\mathrm{NO}_{3}$ after sequence bath reactor with aerobic granular activated sludge (GSBR) anaerobic phase.

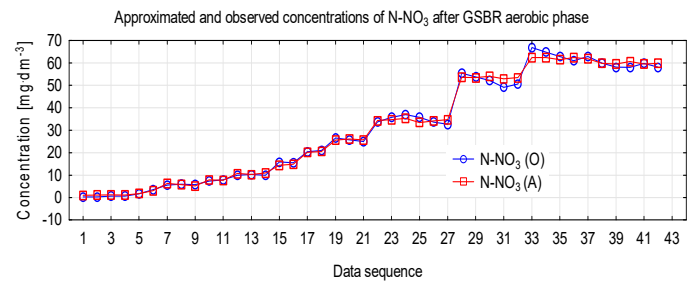

Figure 2. Approximated and observed concentrations of $\mathrm{N}-\mathrm{NO}_{3}$ after sequence bath reactor with aerobic granular activated sludge (GSBR) aerobic phase.

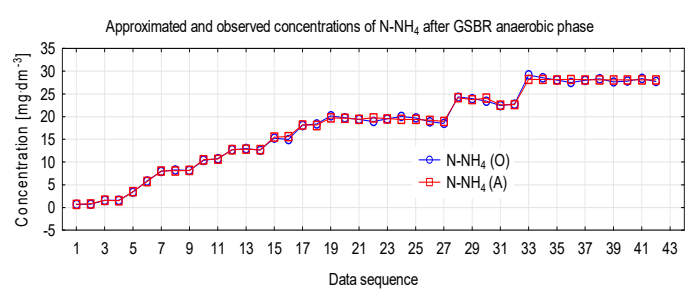

Figure 3. Approximated and observed concentrations of $\mathrm{N}-\mathrm{NH}_{4}$ after sequence bath reactor with aerobic granular activated sludge (GSBR) anaerobic phase. 


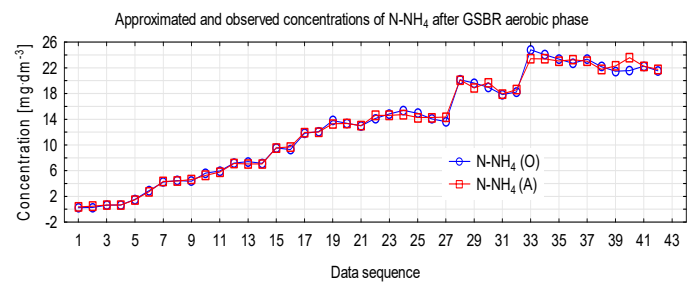

Figure 4. Approximated and observed concentrations of $\mathrm{N}-\mathrm{NH}_{4}$ after sequence bath reactor with aerobic granular activated sludge (GSBR) aerobic phase.

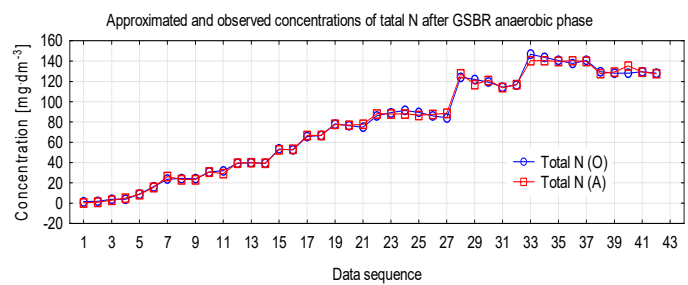

Figure 5. Approximated and observed concentrations of total $\mathrm{N}$ after sequence bath reactor with aerobic granular activated sludge (GSBR) anaerobic phase.

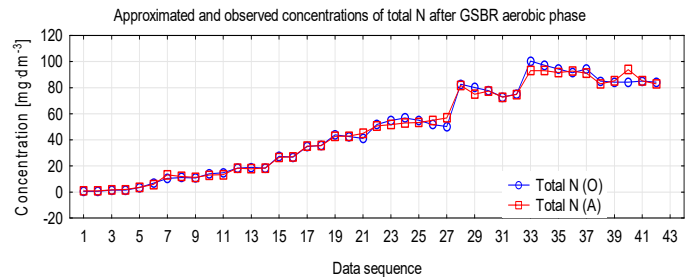

Figure 6. Approximated and observed concentrations of total $\mathrm{N}$ after GSBR aerobic phase.

\section{Discussion}

A similar match between observed and approximated values of $\mathrm{N}-\mathrm{NH}_{4}(\mathrm{r}=0.9987)$ was recived by Zaghloul et al. [5]. Model presented by Zaghloul and others [5] contained 8 variables in the input layer, which were describing wastewater parameters $\left(\mathrm{N}-\mathrm{NH}_{4}, \mathrm{PO}^{3-}\right.$ and $\left.\mathrm{COD}\right)$ activated sludge technological parameters (organic loading rate, hydraulic retention time) and GSBR technological parameters including aeration time, settling time, and exchange ratio. Authors emphasizes that modeling of GSBR process phases in single model is problematical due to different mass transfer in aerobic and anaerobic conditions. The model presented in this work differs in the number of input variables and did not include parameters describing the amount of carbon in treated wastewater. Nevertheless, approximation results observed for $\mathrm{N}-\mathrm{NH}_{4}$ were similar in both models.

\section{Conclusions}

1. The values approximated by the ANN models showed a good fit to the values observed both in the anaerobic phase and in the aerobic phase of the GSBR reactor. Prediction quality for presented models varied form $r=0.996$ to $r=0.999$.

2. The BOD load in the wastewater inflowing to GSBR reactor had the greatest impact on the calculation algorithms approximating concentration of $\mathrm{N}-\mathrm{NO}_{3}$ in the anaerobic and aerobic phases.

3. The concentration of $\mathrm{N}-N H_{4}$ and total nitrogen showed the greatest sensitivity to the concentration of those nitrogen forms in wastewater. 
4. Activated sludge technological parameters did not affect significantly ANN algorithm calculation in anaerobic and aerobic phase. This phenomenon could be resulting from aerobic granular sludge tolerance to toxic agents in wastewater, in this case, oily substances. Therefore, influence of oily substances on wastewater treatment process has not been observed during experiment.

Authors contributions: P.O. conceived and designed the experiments; P.O. performed the experiments; P.O. analyzed the data; J.S.-S. contributed reagents/materials/analysis tools; P.O. and J.S.-S. wrote the paper.

Conflicts of Interest: The authors declare no conflict of interest.

\section{References}

1. Corsino, S.F.; Capodici, M.; Morici, C.; Torregrossa M. Simltaneous nitrition-denitrition for the treatment of high-strength nitrogen in hypersaline wastewater by aerobic granular sludge. Water Res. 2016, 88, 329336, doi:10.1016/j.watres.2015.10.041

2. Adav, S.S.; Lee, D-J.; Show, K-Y.; Tay, J-H. Aerobic granular sludge: Recent advances. Biotechnol. Adv. 2008, 26, 411-423, doi:10.1016/j.biotechadv.2008.05.002

3. Beaten, J.E.; van Loosdrecht, M.C.M.; Volcke E.I.P. Modelling aerobic granular sludge reactors through apparent half-saturation coefficients. Water Res. 2018, 146, 134-145, doi:10.1016/j.watres.2018.09.025

4. Ofman, P.; Skoczko, I. PAH removal effectiveness comparison from hydraulic fracturing model wastewater in SBR reactors with granular and flocked activated sludge. Desalin. Water Treat. 2018, 134, 41-51, doi:10.5004/dwt.2018.22590

5. Zaghloul, M.S.; Hamaza, R.A.; Iorhemen, O.T.; Tay, J.H. Performance prediction of an aerobic granular SBR using modular multilayer artificial neural networks. Sci. Total Environ. 2018, 645, 449-459, doi:10.1016/j.scitotenv.2018.07.140

(C) 2019 by the authors. Licensee MDPI, Basel, Switzerland. This article is an open access article distributed under the terms and conditions of the Creative Commons Attribution (CC BY) license (http://creativecommons.org/licenses/by/4.0/). 